\title{
Impact of vitamin D and vitamin D receptor Taql polymorphism in primary human myoblasts
}

\author{
Amarjit Saini ${ }^{1,2}$, Linda Björkhem-Bergman ${ }^{3}$, Johan Boström ${ }^{1,2}$, Mats Lilja ${ }^{1,2}$, Michael Melin ${ }^{1,2,4}$, Karl Olsson $^{1,2}$, \\ Lena Ekström ${ }^{5}$, Peter Bergman ${ }^{6}$, Mikael Altun ${ }^{1,2, *}$, Eric Rullman ${ }^{1,2,4, *}$ and Thomas Gustafsson ${ }^{1,2, *}$ \\ 1Division of Clinical Physiology, Department of Laboratory Medicine, Karolinska Institutet, Karolinska University Hospital, Stockholm, Sweden \\ 2Unit of Clinical Physiology, Karolinska University Hospital, Stockholm, Sweden \\ ${ }^{3}$ Division of Clinical Geriatrics, Departments of Neurobiology, Care Sciences and Neurobiology, Karolinska Institutet, Stockholm, Sweden \\ ${ }^{4}$ Unit of Cardiology, Karolinska University Hospital, Stockholm, Sweden \\ ${ }^{5}$ Division of Clinical Pharmacology, Department of Laboratory Medicine, Karolinska Institutet, Stockholm, Sweden \\ ${ }^{6}$ Division of Clinical Microbiology, Department of Laboratory Medicine, Karolinska Institutet, Stockholm, Sweden
}

Correspondence should be addressed to A Saini: Amarjit.Saini@ki.se

*(M Altun, E Rullman and T Gustafsson contributed equally to this work)

\begin{abstract}
The CC genotype of the vitamin D receptor (VDR) polymorphism Taql rs731236 has previously been associated with a higher risk of developing myopathy compared to TT carriers. However, the mechanistic role of this polymorphism in skeletal muscle is not well defined. The effects of vitamin D on patients genotyped for the VDR polymorphism Taql rs731236, comparing CC and TT carriers were evaluated. Primary human myoblasts isolated from 4 CC carriers were compared with myoblasts isolated from four TT carriers and treated with vitamin $\mathrm{D}$ in vitro. A dose-dependent inhibitory effect on myoblast proliferation and differentiation was observed concurrent with modifications of key myogenic regulatory factors. RNA sequencing revealed a vitamin D dose-response gene signature enriched with a higher number of VDR-responsive elements (VDREs) per gene. Interestingly, the greater the expression of muscle differentiation markers in myoblasts, the more pronounced was the vitamin D-mediated response to suppress genes associated with myogenic fusion and myotube formation. This novel finding provides a mechanistic explanation to the inconsistency regarding previous reports of the role of vitamin $D$ in myoblast differentiation. No effects in myoblast proliferation, differentiation or gene expression were related to CC Vs TT carriers. Our findings suggest that the VDR polymorphism Taql rs 731236 comparing CC vs TT carriers did not influence the effects of vitamin $\mathrm{D}$ on primary human myoblasts and that vitamin $\mathrm{D}$ inhibits myoblast proliferation and differentiation through key regulators of cell cycle progression. Future studies need to employ strategies to identify the primary responses of vitamin $D$ that drive the cellular response towards quiescence.
\end{abstract}

\section{Key Words}

- Vitamin D

- RNA sequencing

- proliferation

- differentiation

- myoblast

\section{Introduction}

Adequate vitamin D concentrations play a key role in skeletal health. Although there is no agreement on optimal plasma levels of vitamin $\mathrm{D}$, it is apparent that blood precursor/pre-hormone 25-hydroxyvitamin D (25(OH)D) levels are often below recommended ranges for the general population. Prevention of the well-described disorders of rickets and osteomalacia relating to vitamin D levels are clearly important, but vitamin D deficiency (often defined as plasma $25(\mathrm{OH}) \mathrm{D}<25 \mathrm{nmol} / \mathrm{L}$ ) may also lead to muscle weakness, falls and fragility fractures in https://ec.bioscientifica.com

https://doi.org/10.1530/EC-19-0194 (c) 2019 The authors Published by Bioscientifica Ltd

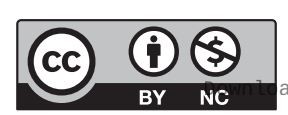

This work is licensed under a Creative Commons Attribution-NonCommercial 4.0 International License. ded from Bioscientifica.com at 04/26/2023 11:01:33AM 
older people (1). These are highly significant public health issues in terms of morbidity, quality of life and costs to health services. vitamin D status has been proposed as an independent indicator of frailty and mortality (2). Several observational studies have evaluated the role of vitamin D on skeletal muscle. Plasma concentrations $<20 \mathrm{ng} / \mathrm{mL}$ $(50 \mathrm{nmol} / \mathrm{L})$ for $25-\mathrm{OHD}$ are associated with increased risk of musculoskeletal pain (3), especially in patients with fibromyalgia (4) and has also been linked to an increased risk of statin-induced myalgia in two observational studies $(5,6)$. It has been shown that old people and patients with $25-\mathrm{OHD}$ concentrations of $46.3 \pm 9.5 \mathrm{nmol} / \mathrm{L}$ have gained muscle strength when treated with vitamin $\mathrm{D}$ $(7,8)$. Nevertheless, a number of studies have failed to demonstrate an effect of vitamin $\mathrm{D}$ on physical performance $(9,10)$, a matter that is still controversial (11). We have previously reported that vitamin D serum levels at $25-\mathrm{OHD}<50 \mathrm{nmol} / \mathrm{L}$ are associated with increased risk of statin-induced myopathy (12).

Vitamin $\mathrm{D}$ signalling occurs through binding by $1,25(\mathrm{OH})_{2} \mathrm{D}_{3}$, the active form of vitamin $\mathrm{D}$, to its cognate nuclear vitamin $\mathrm{D}$ receptor (VDR). A heterodimer, formed with retinoid $\mathrm{X}$ receptor (RXR), then binds specific genomic sequences (vitamin $\mathrm{D}$ response elements or VDREs) acting to influence gene transcription (13). VDREs have been found in several hundred genes that are involved in a multitude of fundamental cellular processes $(14,15,16,17,18,19,20,21)$. VDR-knockout mice provided evidence for a direct role of this steroid receptor in skeletal muscle. Endo and co-workers demonstrated that myofibres in VDR-null mutant mice were smaller than those in WT mice (22). Moreover, skeletal muscles from 3-week-old mice lacking VDR display an abnormally elevated expression of myogenic differentiation factors that are important for the regulation of the satellite cell (SC) niche, i.e. myoblast stem cells (22). Adult skeletal muscle is terminally differentiated and in response to injury, a local pool of SCs situated beneath the basal lamina surrounding each myofibre proliferate (23). Their progeny myoblasts migrate to the sites of damage, and then undergo differentiation leading to their fusion either with each other to form nascent myofibres or with existing myofibres (23). Support for the SC niche as a target for vitamin $\mathrm{D}$ has been demonstrated in our recent report showing VDR to be predominantly located in the muscle precursor cells and not in the skeletal muscle fibre itself (16). Moreover, vitamin $\mathrm{D}\left(1,25(\mathrm{OH})_{2} \mathrm{D}_{3}\right)$ administration to this population induced a pronounced inhibitory effect on myoblast proliferation and differentiation suggesting it is important in maintaining the SC pool (16).
The VDR gene has multiple polymorphisms, most of which are identified by a bi-allelic variation in the restriction endonuclease site (e.g., FokI, BsmI, Apa and TaqI), and these polymorphisms have been suggested to be among the targets of interest explaining inter-individual differences in skeletal muscle mass and strength (24). We have previously shown that patients homozygous for the C-allele of the VDR polymorphism TaqI rs731236 had four times increased risk of developing myopathy than those homozygous for the T allele (12). However, there is a lack of basic understanding on how various VDR polymorphisms influence key regulating processes and pathways in skeletal muscle.

Since VDR seems to be predominantly located to muscle precursor cells in human skeletal muscle (16), it seems rational to study the biological importance of the VDR polymorphism in myoblasts. In the present study, our aim was to compare the effects of the VDR TaqI genotype on vitamin $\mathrm{D}$ molecular signalling by conducting studies on isolated primary human myoblasts from CC and TT individuals. Thus, we hypothesised that vitamin D-mediated effects on primary human myoblasts should be less pronounced with the CC genotype, as a consequence of altered vitamin D-regulated molecular signalling. By analysing the cellular response in relation to altered gene expression using RNA sequencing (RNA-seq), we also aimed to provide further insight into vitamin D-mediated effects on primary human myoblasts.

\section{Materials and methods}

\section{Study participants and ethical approval}

Individuals were recruited from a previous vitamin $\mathrm{D}$ study in which all participants had been genotyped for VDR TaqI polymorphism. Eight individuals were included in the study, two males and six females, mean age $57.4 \pm 13.6$ years of which four had CC genotype and four TT genotype. All participants had a BMI between 19 and $25 \mathrm{~kg} / \mathrm{m}^{2}$. The 25-hydroxyvitamin $\mathrm{D}$ levels varied between 40 and $81 \mathrm{nmol} / \mathrm{L}$, and there was no statistically significant difference between the two groups; average 25 -hydroxyvitamin D levels were $61 \mathrm{nmol} / \mathrm{L}$ compared to $62 \mathrm{nmol} / \mathrm{L}$. All participants had an increased susceptibility to respiratory tract infections. The diagnostic entities included IgA- and IgG-subclass deficiency. No statin treatment was allowed but other medications such as antihypertensive and anti-depressant drugs were permitted. The genotyping had been performed using TaqMan

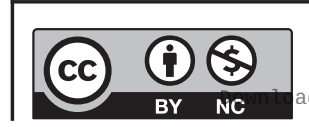

This work is licensed under a Creative Commons Attribution-NonCommercial 4.0 International License. ded from Bioscientifica.com at 04/26/2023 11:01:33AM 
genotyping assay (C_2404008_10; Applied Biosystems) for the VDR polymorphism (rs731236 also referred to as TaqI) to identify individuals homozygous for TT or CC (25). Both groups consisted of one male and three females, TT mean age $55.5 \pm 11.8$ years; CC $59.3 \pm 16.8$ years.

\section{Ethical statement}

Written informed consent was obtained from all participates before any study-related procedure was performed. The study was approved by the Regional Ethical Review Board in Stockholm (Dnr. 2015/210-31) and was performed in accordance with the Declaration of Helsinki.

\section{Skeletal muscle biopsy}

Resting skeletal muscle biopsies were collected from the vastus lateralis muscle via the percutaneous needle biopsy technique as described previously $(26,27)$. After sterilisation (chlorhexidine alcohol cutaneous solution, $5 \mathrm{mg} / \mathrm{mL}$; Fresenius Kabi) and local anaesthesia (Carbocain, $10 \mathrm{mg} / \mathrm{mL}$; Astra Zeneca), a small insertion in the skin was made with a scalpel. The muscle biopsy was obtained using the Bergström needle to penetrate the muscular fascia and connective tissue. Biopsy material for myoblast isolation was immediately put in sterile PBS (Gibco Invitrogen, Life Technologies) containing $1 \%$ antibiotic-antimycotic (ABAM) (Gibco Invitrogen, Life Technologies), whereas muscle tissue for protein and RNA analysis was snap frozen in isopentane and stored at $-80^{\circ} \mathrm{C}$.

\section{Myoblast isolation and cell culture}

All reagents used for myoblast isolation as well as human myoblast and monocytic cell culture were from Gibco Invitrogen, Life Technologies. Myoblasts were extracted from fresh muscle tissue as previously described (26). Immediately after the biopsy procedure, approximately $100 \mathrm{mg}$ of muscle tissue were placed in PBS solution containing 1\% ABAM and incubated at $4^{\circ} \mathrm{C}$ overnight. The next day, the muscle biopsy was incubated in $5 \mathrm{~mL}$ TrypLE Express Enzyme $(1 \times)$ at $37^{\circ} \mathrm{C}, 5 \% \mathrm{CO}_{2}$ with gentle agitation for $20 \mathrm{~min}$. Undigested tissue was allowed to settle for $5 \mathrm{~min}$ at room temperature, and the supernatant containing the myogenic cells was collected in $5 \mathrm{~mL}$ DMEM-F12 GlutaMAX, containing 20\% fetal bovine serum (FBS) and 1\% ABAM. Digestion of the slurry was repeated twice. Isolated myoblasts were cultured in
DMEM-F12 GlutaMAX containing 20\% FBS and 1\% ABAM (high-serum media) at $37^{\circ} \mathrm{C}, 5 \% \mathrm{CO}_{2}$. Culture dishes were coated with collagen I (collagen I, bovine $5 \mathrm{mg} / \mathrm{mL}$ ) diluted to a final concentration of $50 \mu \mathrm{g} / \mathrm{mL}$ in $0.02 \mathrm{M}$ acetic acid according to the manufacturer's instructions. Myoblasts were taken through serial passages to increase cell numbers before experimentation. All myoblasts were used for experimentation at passages 4-5. For experimentation, myoblasts were harvested and transferred to collagen I-coated six-well plates at a density of $1 \times 10^{5}$ cells per well and allowed to settle in high-serum media for at least $24 \mathrm{~h}$ before experimentation. Myoblast differentiation was promoted by substituting the proliferation media with DMEM-F12 GlutaMAX containing 2\% FBS and 1\% ABAM (low-serum media).

\section{Immunomagnetic cell separation}

Enrichment of the cell population for myogenic cells was accomplished by a combination of pre-plating and magnetic-activated cell sorting (MACS) separation as has previously been reported to produce a high yield of myogenic cells (28). MACS separation of myogenic and non-myogenic cells was carried out as previously described for human muscle-derived cells (16), with some modifications. Muscle-derived cells plated in T75 flasks were incubated with a mouse antihuman antibody for CD56 (MY31; BD Biosciences) dissolved in DMEM-F12 GlutaMAX for $30 \mathrm{~min}$ at $37^{\circ} \mathrm{C}, 5 \% \mathrm{CO}_{2}$. Cells were subsequently pelleted and resuspended in PBS containing $0.1 \%$ FBS and antimouse IgG microbeads (Miltenyi Biotech) according to the manufacturer's instructions. Cell suspension was incubated in the dark at $4^{\circ} \mathrm{C}$ for 15 min before being rinsed with PBS containing 0.1\% FBS and repelleted. Cells were magnetically separated using a miniMACS magnet and LS columns (Miltenyi Biotech). The cells that were bound to the anti-CD56 microbead complex were maintained in the column and constituted the positive (myogenic) fraction of cells. This fraction was subsequently plated and cultured as described earlier.

\section{Validation of myogenic origin}

At the time of plating cells for experimentation, a fraction of myoblasts was collected for confirmation of myogenic origin. Cells were spun down onto a cover glass for subsequent immunofluorescent staining for the myogenic marker desmin (ab15200; Abcam). The fraction of desmin-positive cells in the cell population was analysed by dividing with the total number of nuclei

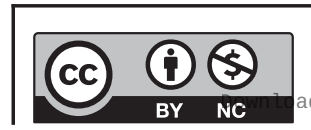

This work is licensed under a Creative Commons Attribution-NonCommercial 4.0 International License. ded from Bioscientifica.com at 04/26/2023 11:01:33AM 
counterstained with 4',6-diamidino-2-phenylindole di-hydrochloride (Molecular Probes) within each field. In the current study, 98\% $( \pm 1.7)$ of sub-confluent myoblasts were positive for desmin (Supplementary Figure 2, see section on supplementary data given at the end of this article).

\section{Cell proliferation analysis}

\section{EdU}

For EdU incorporation, mitotic G2 and cell viability analysis, myoblasts were seeded in 96-well glass bottom microplates (BD Falcon) at a density $1.5 \times 10^{3}$ cells/well. After $48 \mathrm{~h}$, media was replaced containing 0.01, 1, $100 \mathrm{nM}$ $1 \alpha, 25(\mathrm{OH})_{2} \mathrm{D}_{3}$ (vitamin $\mathrm{D}$; Sigma-Aldrich) or vehicle (dimethyl sulfoxide) (Sigma-Aldrich) in either low- or highserum media and stimulated for 24 and $48 \mathrm{~h}$. Following stimulation myoblasts were incubated with $10 \mu \mathrm{M}$ EdU 60 min prior to fixation in $4 \%$ paraformaldehyde (PFA) in PBS for $15 \mathrm{~min}$ at RT. Fixed cells were washed with PBS and permeabilised with $0.3 \%$ Triton X-100 in PBS (PBST) and then incubated $30 \mathrm{~min}$ at room temperature with $2 \mu \mathrm{M}$ Alexa 647-conjugated Azide, $10 \mathrm{mM}$ ascorbic acid, $2.5 \mathrm{mM} \mathrm{CuSo}_{4}$ in PBS. DAPI staining was performed by a 1 -h incubation at $4^{\circ} \mathrm{C}$ with $10 \mu \mathrm{g} / \mathrm{mL}$ RNAse, followed by $10 \mathrm{~min}$ of $1 \mu \mathrm{g} / \mathrm{mL}$ DAPI in PBS at room temperature. All plates were imaged on an ImageXpress imaging system (Molecular Devices) at 10x magnification, and segmentation of nuclei and measurement of fluorescence intensities were performed in CellProfiler (29).

\section{Cell stimulation protocol}

For analysis of the effects of vitamin D metabolites on myoblast gene expression and differentiation, high-serum media were changed to low-serum media containing vitamin D, $0.01,1,100 \mathrm{nM}$ or DMSO alone. After $24 \mathrm{~h}$ of stimulation, RNA was collected.

\section{RNA extraction and sequencing}

Total RNA was prepared by the TRIzol method (Invitrogen, Life Technologies) and quantified spectrophotometrically by absorbance at $260 \mathrm{~nm}$. One $\mu \mathrm{g}$ of total RNA was reverse transcribed by Superscript reverse transcriptase (Life Technologies) using random hexamer primers (Roche Diagnostics) in a total volume of $20 \mu \mathrm{L}$. The preparation of the cDNA library and the RNA sequencing was performed by the core National Genomics Infrastructure (NGI) facility Science for Life at Karolinska Institutet
(Solna, Sweden). The cDNA originating from the RNA fragments were paired and sequenced using the highthroughput sequencing platform of Illumina HiSeq. 3000 , and $6 \mathrm{G}$ reads per sample were obtained on average. Our sequencing data have been submitted to the Gene Expression Omnibus (GEO) database and assigned the accession number as 'Pending'.

The expression levels of each sample were normalised as Transcripts Per Kilobase Per Million (TPKM) by dividing the read count of each transcript model with its length and scaling the total per sample to one million. Differential gene expression analysis was performed on the mapped read counts using the edgeR package in R (30), correcting for common, trended and tagwise disparity with the formula log TPKM 0+Genotype+Genotype:log (Concentration). Baseline differences between cells from different genotypes and tSNE-based clusters were tested for differential expression using moderated t-statistics in edgeR. The analysis rendered $P$ values, logFC differences between groups and FDR values. Data-driven, clustering of observations was explored using the Rtsne package (31). Presence of VDR-responsive elements was identified using Gene Transcription Regulation Database V18.06 identifying the number of VDR-responsive elements 5 and $10 \mathrm{kbp}$ upstream of protein-coding genes using a meta-analysis of Chip-seq experiments (32).

\section{Quantitative polymerase chain reaction (qPCR) for target genes}

To validate RNA sequencing transcripts, mRNA quantitation was performed on an ABI-PRISM7700 Sequence Detector (PerkinElmer, Applied Biosystems). Primer and probe for GAPDH (glyceraldehyde-3-phosphate dehydrogenase), VDR and CYP24A1, were ordered as assay on demand (GAPDH, 4352934E; VDR, Hs00172113_m1; CYP24A1, Hs00167999_m1; PerkinElmer, Applied Biosystems). Target gene expression was subsequently reported as a ratio relative to the respective reference genes by the $2^{-\Delta \Delta \mathrm{CT}}$ formula.

\section{Statistical analysis}

Statistically significant differences when comparing more than two groups were evaluated by the use of a one-way ANOVA or one-way repeated-measures ANOVA. Tukey's post hoc test was used to locate the differences in mean values. Differences were considered significant at $P<0.05$. Data are presented as the mean \pm S.E.M. unless stated otherwise. 


\section{Results and analysis}

\section{The VDR Taql genotype has no impact on vitamin} D-mediated inhibition of proliferation

The effect of vitamin D on myoblast proliferation was analysed by EdU incorporation, an indicator of actively proliferating cells, after 48 -h exposure to $0.01 \mathrm{nM}, 1 \mathrm{nM}$ and $100 \mathrm{nM}$ vitamin D or DMSO control under high serum (20\% FCS) conditions. Overall, there was a dosedependent block following vitamin $\mathrm{D}$ treatment on EdU incorporation in muscle cells compared with DMSO control. This was significantly different $(P<0.001)$ from all other treatments at the highest dose of vitamin $D$ administered (Fig. 1A). A similar trend was observed for change in percentage of cells in G2 phase, the stage preceding mitosis and following the completion of $\mathrm{S}$ phase. Addition of vitamin $\mathrm{D}$ at the highest dose compared to all other treatments resulted in a significant $(P<0.001)$ decrease in the population of myoblasts to potentially undergo mitosis (Fig. 1B). The consequence of fewer cells entering mitosis was that after $48 \mathrm{~h}$ with $100 \mathrm{nM}$ vitamin $\mathrm{D}$, there was a decline in the population of cells that was significant $(P<0.05)$ only for the TT genotype $(-133 \pm 69$ CC; $-161 \pm 64$ TT) compared to control (Fig. 1C). In each case, the comparison of genotypes on the percentage change for EdU positive, G2 phase and cell count was that they were similar with respect to genotype. The VDR TaqI rs731236 polymorphism had no impact on cell cycle progression within CC and TT carriers neither in the presence nor absence of vitamin $\mathrm{D}$.

\section{Baseline characterisation of CC and TT-derived myoblasts}

Baseline differential expression analysis of RNA-seq data did not identify any genes that were differentially expressed between cells with different genotypes, CC or TT (Supplementary Table 1). Moreover, data-driven unsupervised analysis of gene expression at baseline, that is, comparison of CC versus TT cells without addition of vitamin D did not reveal any clustering related to genotype, sex or age of the donor of the myoblasts (Fig. 1D).

There were no transcripts (including transcripts assessed by qPCR) where the response was significantly different in CC versus TT-derived cells, neither with regards to magnitude nor direction with vitamin $\mathrm{D}$ stimulation (Supplementary Figures 1A and B). Moreover, vitamin D induced a dose-dependent increase in VDR and CYP24A1 without any significant differences between CC and TT myoblasts (Fig. 1E and F). Thus, CC and TT cells responded to vitamin $\mathrm{D}$ treatment in a similar manner. The analysis of dose-response of the transcriptome to vitamin $\mathrm{D}$ revealed 1300 transcripts with vitamin D response in both CCand TT-derived myoblasts (Fig. 2A, B and Supplementary Table 1). Gene-ontology analysis using DAVID and GSEA revealed enrichment of gene ontologies in myoblasts following 24-h stimulation were primarily related to cell proliferation or differentiation, such as skeletal myofibril assembly, DNA strand elongation, mitotic chromosome condensation, skeletal muscle adaptation and protein localisation to kinetochore (Fig. 2C). Notably, among the most downregulated transcripts identified were Cyclin A2

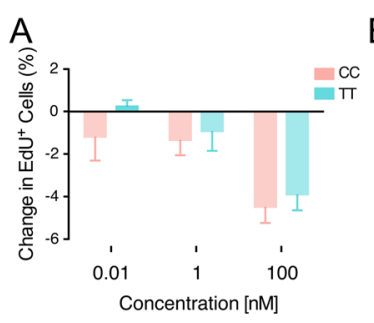

$\mathrm{D}$

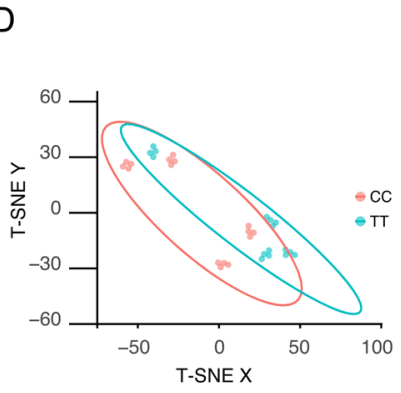

E VDR

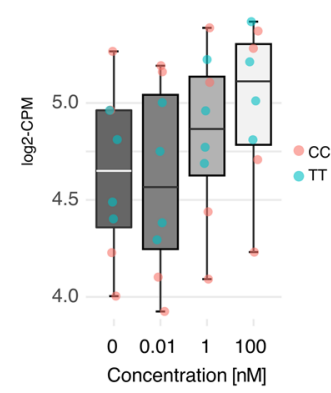

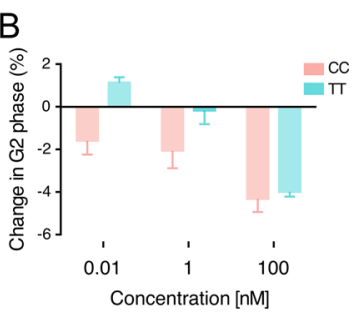

E VDR

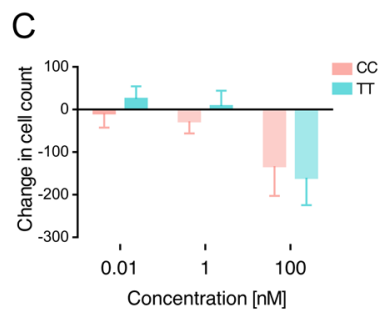

F CYP24A1

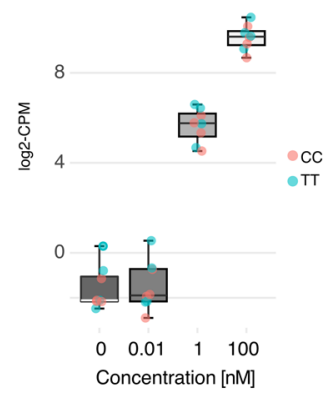

\section{Figure 1}

Using high-throughput imaging analysis, primary human muscle cells with the Taq1 polymorphism were stained and imaged for DAPI and EdU incorporation after 48-h exposure to DMSO alone or $0.01 \mathrm{nM}, 1 \mathrm{nM}$ and $100 \mathrm{nM} 1,25(\mathrm{OH})_{2} \mathrm{D}_{3}$. Data are presented for change in total cell count (A), change in the percentage incorporation of EdU-positive cells (B) and change in the percentage of cells that are $G 2$ positive (C), as the mean \pm S.E.M. TT versus CC. (D) Unsupervised clustering using tSNE of gene expression of all cells and all used dosages of vitamin. ( $E$ and $F$ ) VDR and CYP24A1 expression following various dosages of vitamin $D$. https://ec.bioscientifica.com https://doi.org/10.1530/EC-19-0194 (c) 2019 The authors Published by Bioscientifica Ltd

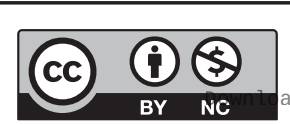

This work is licensed under a Creative Commons Attribution-NonCommercial 4.0 International License. ded from Bioscientifica.com at 04/26/2023 11:01:33AM via free access 
A

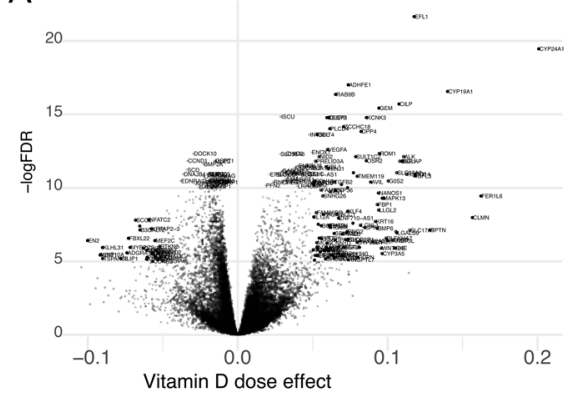

C

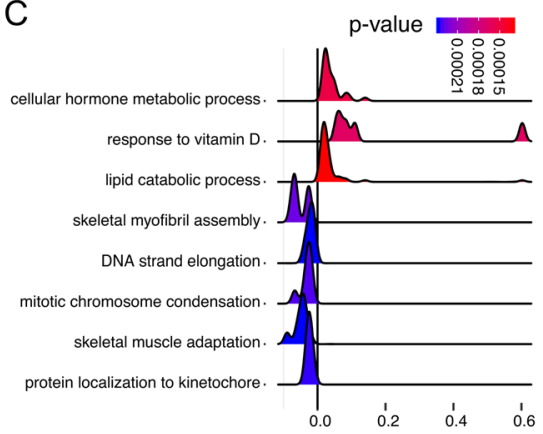

E

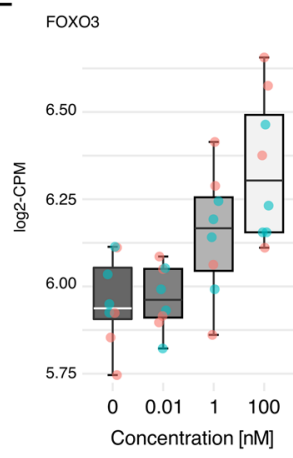

$\mathrm{F}$

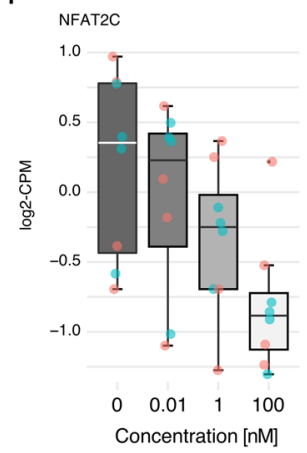

B

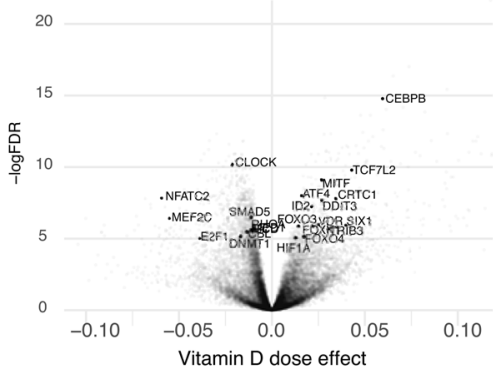

D

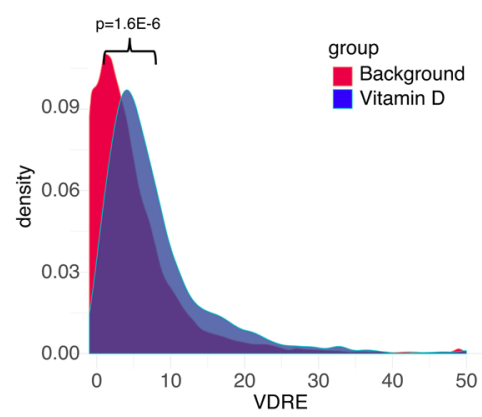

G

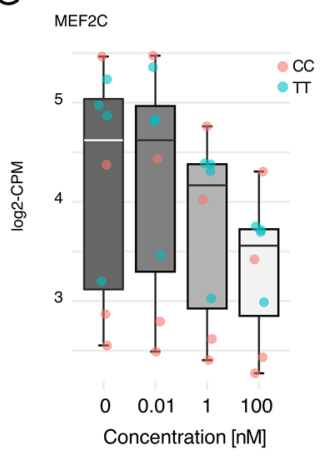

\section{Figure 2}

(A) Volcano plot illustrating genes differentially expressed in a dose-dependent manner upon stimulation with vitamin D. (B) Volcano plot highlighting transcription factors differentially expressed upon vitamin D stimulation. (C) Illustration of dose-dependent changes in gene expression of the top-most significant biological functions identified using gene-set enrichment analysis (GSEA). (D) Distribution of the number of VDR-responsive elements $\leq 10 \mathrm{kbp}$ upstream of vitamin $D$ dose-responsive genes (green), versus non-differentially expressed genes (red). (E) Boxplots of selected differentially expressed transcription factors illustrating vitamin $D$ dose-response for three selected genes: FOXO3, NFAT2 and MEF2. and D1, associated with cell cycle progression (Fig. 2A). On the regulatory side, a dose-dependent increase in the RNA expression of FOXO3, previously associated with cell quiescence (33), was observed following vitamin D stimulation. Moreover, NFAT2C which encodes for the NFAT2C transcription factor and controlling myoblast fusion at a specific stage of myogenesis $(34,35)$ and MEF2C (Fig. 2B, E, F and G) with a similar function (36) decreased with vitamin $\mathrm{D}$ addition.

\section{Vitamin D dose-response signature for VDREs}

The vitamin D dose-responsive signature was analysed for enrichment of VDREs using the hypergeometrical test. On average there were 6.5 VDR-responsive elements within $10 \mathrm{~kb}$ upstream of the genes in the signature compared with 6.3 for all investigated genes $(P<0.01)$, and there was a more pronounced overrepresentation of VDREs among genes with a negative dose-response with 7.8 VDR upstream elements on average (Fig. 2D).

Overall, the human myoblast vitamin D doseresponse RNA-seq signature was largely consistent with previous analyses from our laboratory of high-dose vitamin D treatment analysed on U133 microarray platform (16), where 94/174 (55\%, $P<0.001)$ of the genes with a positive dose-response to vitamin $\mathrm{D}$ were also upregulated after high-dose treatment and analysed on the U133 microarray platform and 17/93 (18\%, P<0.01) of the genes with a negative dose-response to vitamin $D$ were overlapping (Supplementary Table 1).

\section{Myoblast differentiation determines the vitamin D response}

The results of the tSNE clustering (Fig. 1D) showed two distinct clusters of observations with CC and TT 
genotypes equally represented in each cluster, that is, genotype had no impact on grouping. Thus a differential analysis of the clustering was performed in order to define what genes distinguished the two clusters: First, differential expression analysis of the clusters at baseline vehicle control confirmed that the distinguishing feature was the degree of expression of differentiation markers in myoblasts. A large number of genes associated with mature muscle fibres (Fig. 3A and B), such as TNNT, were only detected in the cluster of myoblasts expressing a more differentiated gene signature. This notion was further corroborated by gene-ontology analysis where biological processes related with muscle differentiation were 25-fold enriched (FDR 3E-4) (Fig. 3C). The VDR gene was not differentially expressed between the two clusters (log-fold change -0.06, FDR 0.91), and there was no enrichment of VDREs in the genes distinguishing the two myoblast clusters. There was a pronounced difference between the two clusters with regard to the response to vitamin D; 567 were differentially expressed in the cell cluster of myoblasts expressing a more differentiated signature versus 327 in the cell cluster of myoblasts expressing a less differentiated signature (Fig. 4A and Supplementary Table 2). In particular, genes associated with muscle differentiation (differing also between the clusters at baseline) were robustly downregulated in a dose-responsive manner in myoblasts with more differentiated profile (Fig. 4B). Several genes associated with muscle differentiation were regulated in opposing directions in the differentiated versus less differentiated cells (Fig. 4B), exemplified by RNA expression of miR-133, MSTN (Myostatin) and RYR1 (Ryanodine receptor 1)
(Fig. 4C, D and E), all known to be more highly expressed in myotubes than myoblasts (37).

\section{Discussion}

Using primary human myoblasts extracted from skeletal muscle biopsies obtained from individuals with VDR TaqI polymorphism (CC and TT carriers), the effects and potential differences of vitamin D molecular signalling in regulating the myoblast response were investigated. Our primary finding is that no effects were observed at the time points investigated for myoblast proliferation, gene signature for differentiation or the overall gene expression profile related to the CC or TT genotype. Similar to previous findings (16), a dose-dependent inhibitory effect on myoblast proliferation and differentiation was observed concurrent with modification of key myogenic regulatory factors. Within the myoblast population, the expression pattern following 24 -h vitamin D stimulation - preceding the events of myoblast fusion/myotube formation - indicated differentiation was not uniform. Interestingly, greater expression of differentiation markers in myoblasts was associated with a more pronounced vitamin D-mediated suppression of genes associated with myogenic fusion and myotube formation.

The VDR TaqI polymorphism CC or TT SNP is a silent SNP located in exon 9 which has been proposed to be linked to gene regulation (38) and previously suggested to be a marker for increased risk of myopathy (12). In the current study, proliferation or the molecular events leading to myogenic differentiation and fusion
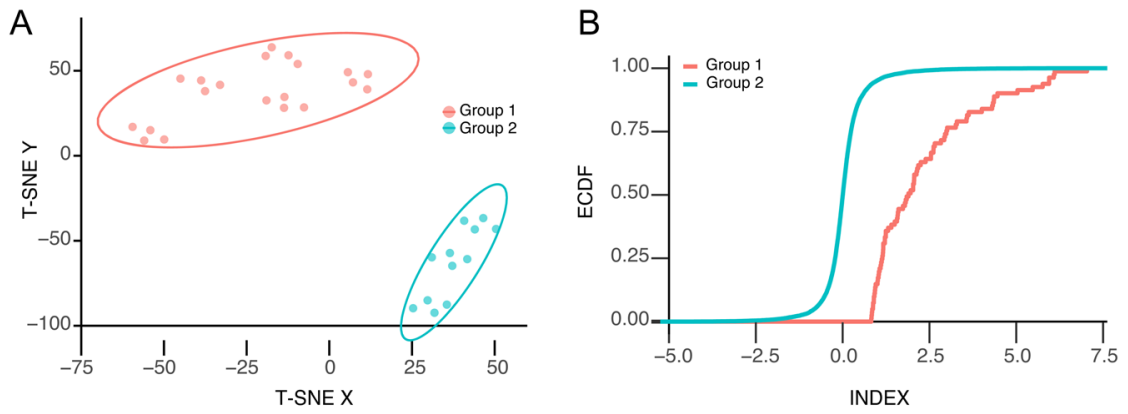

C

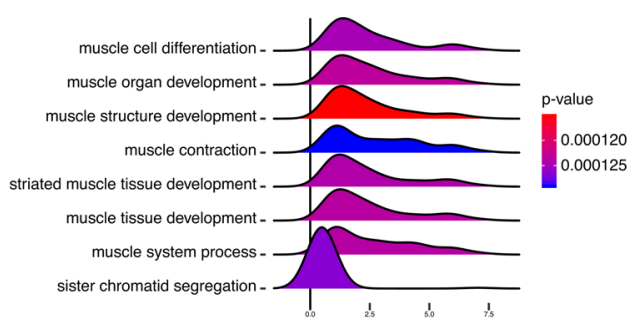

\section{Figure 3}

(A) Unsupervised cluster analysis of the transcriptome using tSNE revealed two distinct groups of cells. (B) Empirical cumulative distribution (ECDF) graph of genes involved in 'muscle differentiation' in the two groups. (C) Differences in gene expression between the two groups illustrated by ridgeplot of using gene-set enrichment analysis (GSEA). 
A

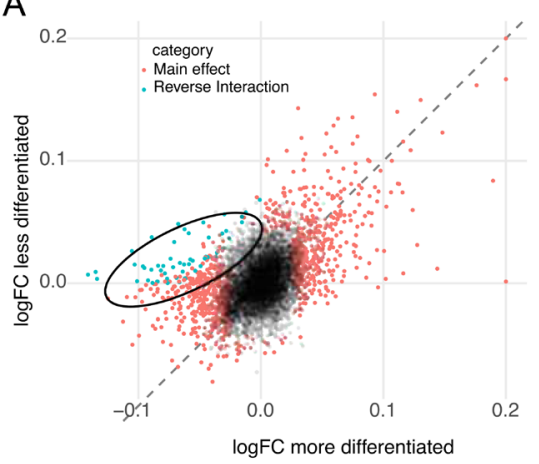

B

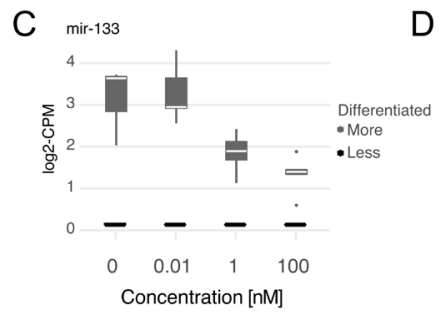

D MSTM

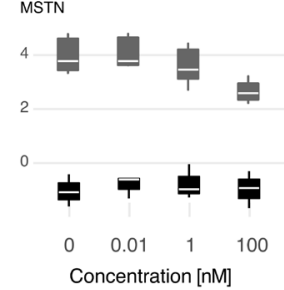

of primary human myoblasts isolated from individuals homozygous for the CC alleles genotype of the VDR polymorphism TaqI rs731236 did not differ following vitamin D treatment compared to myoblasts extracted from individuals homozygous for TT alleles. RNA-seq gene expression profiling also showed no difference, neither at baseline nor when stimulated with vitamin D at various vitamin D concentrations $(0.01,1,100 \mathrm{nM})$. Thus, the TaqI polymorphism alone following 24-h treatment between CC and TT carriers does not seem to alter the gene signature in response to vitamin $\mathrm{D}$ on primary human myoblasts. This was further underlined by observations of a similar mRNA increase in the two most common molecular markers for VDR activation, VDR and CYP24A1.

In addition to evaluating the importance of TaqI rs731236, we aimed to expand our earlier knowledge of vitamin D effects on the SC niche. Consistent with our earlier report (16), the present study revealed a dosedependent block on EdU incorporation in myoblasts and reduced number of G2-positive cells, which occurred in parallel with reduced expression of genes controlling the G1/S restriction point. In fact, two of the most affected genes were Cyclin A2 and D1, both involved in regulating cell cycle progression. By analysing the cellular response in relation to gene expression patterns by bioinformatic analyses, we aimed to provide further depth into our earlier array study. Collectively, our findings demonstrate a reduction of mRNA expression in ontologies relating to cell proliferation and differentiation. Regulation of SC

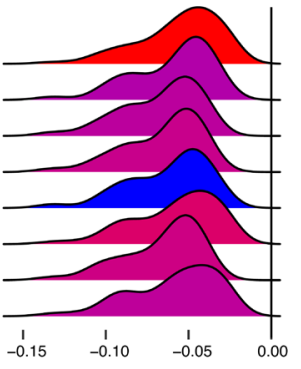

Figure 4

(A) Scatterplot illustrating the relationship between the vitamin $D$ dose-response in the differentiated versus less differentiated cluster of cells. Red denotes genes up or downregulated in a similar fashion in both groups. Encircled blue are a subset of genes, all of which were significantly downregulated upon vitamin $\mathrm{D}$ stimulation in more differentiated cells, but were instead upregulated in cells from the less differentiated cluster (FDR for interaction $<0.05$ ). (B) A ridgeplot illustrating gene-set enrichment analysis (GSEA) of the genes with opposite/ contrasting differential expression pattern in more versus less differentiated cells. Boxplots of genes with a reversed expression pattern in more versus less differentiated cells illustrating a clear anti-differentiation effect of vitamin D where cells with high expression of genes involved in muscle differentiation respond with a robust downregulation of these genes, (C) miR-133, (D) MSTN (Myostatin) and (E) RYR1.

self-renewal and quiescence is tightly regulated and involves reversible exit from the cell cycle and an inhibition of myoblast differentiation. Overall, the current vitamin D dose-response gene signature was consistent with our previous report using the U133 microarray platform (16). The RNA-seq analysis identified key regulatory factors FOXO3, MSTN (Myostatin), NFATC2 and MEF2C among those with dose-dependent changes. It has been shown that FOXO3 is expressed in quiescent cells, required for self-renewal and suggested to promote Notch signalling, a key regulator of SC quiescence (33). Moreover, MSTN is known, through various processes, to inhibit both proliferation and differentiation (39), including induction of Notch signalling and downregulation of the myogenic regulatory factor MYOD1 $(39,40)$. In myoblasts, MSTN has been shown to prevent progression from the G1-to-S phase of the cell cycle (41), consistent with the current observation of vitamin $\mathrm{D}$ treatment to be inversely correlated to a reduction in myoblasts entering the G2 phase. NFATC2 and MEF2C are known to increase throughout the transition from myoblast to myotubes $(34,35,36)$. The genes regulated by NFATC2, in addition to the MEF family, are crucial for myoblasts to differentiate and fuse into multinucleated myotubes (34, 35,36 ). Therefore, downregulation of NFATC2 and MEF2C in combination with increased MSTN and FOXO3/Notch signalling suggests an intricate and co-ordinated network of molecular signalling events/pathways that lead to the inhibition of myoblast fusion/differentiation and return to SC quiescence that is orchestrated by vitamin D.

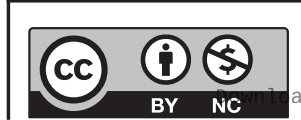

This work is licensed under a Creative Commons Attribution-NonCommercial 4.0 International License. ded from Bioscientifica.com at 04/26/2023 11:01:33AM 
tSNE cluster analysis revealed a cell-cell variance in cell culture for the expression of muscle differentiation factors, with one cluster of a more differentiated and one with a less differentiated gene signature within the myoblast population. Thus, the more pronounced the gene signature towards differentiation in myoblast cells, the more pronounced was the response to vitamin $\mathrm{D}$ treatment. In fact, following vitamin D stimulation, we found a reversed expression pattern in more versus less differentiated cells including genes involved in muscle tissue differentiation and development. Importantly, this novel finding may help explain the previously ambiguous reports of the role of vitamin D in myoblast differentiation, including diverse effects on Mstn expression $(16,42,43)$. The present findings should be taken into consideration when investigating the effects of myoblast differentiation with vitamin D. Although the VDR expression levels did not differ between the two clusters of myoblasts with diverse differentiation gene signatures in the current study, we have previously reported that the VDR expression is augmented at both the mRNA and protein levels following differentiation of myoblasts into myotubes (16). Collectively, even though differences in VDR expression may not explain the diversity of vitamin D responsiveness per se, the current findings add to the expanding regulatory role of vitamin $\mathrm{D}$ in myoblast stem cell decisions that it is enhanced at later stages of myoblast differentiation. Importantly, no differences were observed between genotypes (CC and TT). Both VDR expression and vitamin D-induced CYP24A1 was similar suggesting that VDR molecular signalling was in place in both populations and as shown in Fig. 4, most transcripts responded uni-directionally between the two cell clusters.

Several transcripts were suppressed only in the cluster of cells with a more differentiated gene signature, which mimicked a hypothetical dedifferentiation process, or the ability to suppress the factors needed to push the cells for differentiation and put the cell in a more quiescent state. The pronounced inhibitory effects on cell differentiation by vitamin $\mathrm{D}$ suggest that VDR-dependent signalling pathways presumably need to be downregulated to achieve complete myogenic differentiation. This is in contrast to first reports, mainly originating from $\mathrm{C} 2 \mathrm{C} 12$ cell lines that demonstrate positive vitamin $\mathrm{D}$ effects on myoblast differentiation (44). Since low vitamin D status $(<50 \mathrm{nmol} / \mathrm{L})$ is common in aged individuals and proposed to be an independent indicator of frailty and mortality (2), the conclusion of initial reports from myogenic cells was that vitamin D counteracts muscle wasting and weakness through facilitation of myoblast differentiation and repair. The current study and our earlier report (16) support that vitamin D could be beneficial for the ageing skeletal muscle but through other mechanisms. Asymmetric divisions are less common in old SCs, which more often undergo symmetric divisions and generate two differentiated myoblast cells. Repeated injury and regeneration seem to exhaust the SC pool including to favour selection of more proliferating clones (45). The net result is a rapid exhaustion of the uncommitted SC pool (45). Moreover, indirectly, the present finding suggests vitamin D to activate Notch, which may be a key event since blunted SC activation in the elderly occurs in parallel with diminished activation of Notch signalling (46), and activation of MAPK/Notch restore myogenic responses that is make them more similar to SCs of young adults (47). Thus, vitamin D may be of importance in maintaining the SC pool and consequent regulation of myoblast skeletal muscle regeneration/repair $(46,47)$. Future studies should aim to investigate the potential role of vitamin D and vitamin D status in the maintenance of SC populations.

With the current study design, it was not possible to scale down plausible genes that are regulated primarily through VDR signalling against those related to secondary effects. A more fruitful strategy in this situation may be to shorten the time of exposure to vitamin D so that only the primary targets of vitamin D signalling are upregulated. Indeed, the vitamin D dose-response signature consisted of genes that were enriched for predicted VDREs, but this enrichment was small compared with the background (6.3 versus 6.0 VDREs per gene). This further supports the notion of a cascade effect of secondary cellular responses mediated initially by direct VDR-VDRE signalling. A combination of VDR Chromatin Immunoprecipitation (ChIP) sequencing and RNA sequencing would enable a discrimination between the direct effects of the VDR on the transcript of different cell populations and any downstream signalling cascade.

It has been suggested that individuals homozygous for the CC allele genotype of the VDR polymorphism TaqI rs731236 exhibit an altered expression pattern for the VDR $(48,49)$. At the time point investigated, the current study did not have the power to address this question ( $n=4$, per genotype), but no difference was observed at baseline in VDR expression by RNA-seq analysis, and this was confirmed with qPCR (Supplementary Figures $1 \mathrm{~A}$ and $1 \mathrm{~B})$. However, functionally important differences related to polymorphism-associated differences in the expression of VDR were not revealed following vitamin D treatment in the current study. Although, TaqI rs731236

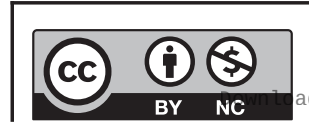

This work is licensed under a Creative Commons Attribution-NonCommercial 4.0 International License. ded from Bioscientifica.com at 04/26/2023 11:01:33AM 
is one of several common genetic variants that has been described in humans, most of which are identified by a bi-allelic variation in a restriction endonuclease site (38), no other plausible SNP was assessed in the current study, and it is possible that other variants may affect the response towards vitamin D. It should however be noted that the present findings reflect only a snapshot of the effect of the VDR between CC and TT carriers for the TaqI polymorphism rs731236 in human myoblasts. The scope of the present study was not to investigate effects on myotubes since we had previously shown (16) that vitamin D suppresses myotube fusion and therefore was relevant to study the effects on the myoblast cell in reference to regulating skeletal muscle regeneration. However, initiating vitamin D treatment at later stages and post fusion (in myotubes) might elicit a different response on expression including between carriers of the CC and TT VDR polymorphism TaqI rs731236. Support for such an idea has been reported in C2C12-differentiated myotubes whereby certain concentrations of vitamin D exhibited an anabolic effect that was absent in predifferentiated murine myoblasts (50). Future studies are therefore required to fully investigate the role of this VDR polymorphism on myoblast including later stages of differentiation.

In the current setting, the CC VDR polymorphism TaqI rs731236 does not influence the vitamin D effects on primary human myoblasts compared with TT carriers that suggest no functional differences in myofibre regeneration of adult human. Nevertheless, the previously established role of vitamin D in the regulation of myoblast proliferation and differentiation processes is corroborated and expanded to preferentially include regulatory events at later stages of myoblast differentiation. Vitamin D modulates the gene expression of key regulators of cell cycle progression and has prominent effects on cells during differentiation. Moreover, as the response to vitamin D stimulation was markedly different depending on myoblast differentiation status, our findings clearly demonstrate the importance of careful characterisation of the phase of differentiation when performing mechanistic studies of stem cells subjected to vitamin D.

\section{Supplementary data}

This is available on the journal website at https://doi.org/10.1530/EC-190194.

The supplementary data is also available on figshare:

Supplementary Table 1: https://doi.org/10.6084/m9.figshare.7732178.v1 Supplementary Figure 1A: https://doi.org/10.6084/m9.figshare.7731827.v2 Supplementary Figure 1B: https://doi.org/10.6084/m9.figshare.7732151.v2 Supplementary Figure 2: https://doi.org/10.6084/m9.figshare.7998221.v1

\section{Declaration of interest}

The authors declare that there is no conflict of interest that could be perceived as prejudicing the impartiality of the research reported.

\section{Funding}

This research did not receive any specific grant from any funding agency in the public, commercial or not-for-profit sector.

Author contribution statement

M Altun, E Rullman and T Gustafsson contributed equally to this work.

\section{References}

1 Spiro A \& Buttriss JL. Vitamin D: an overview of vitamin D status and intake in Europe. Nutrition Bulletin 201439 322-350. (https:// doi.org/10.1111/nbu.12108)

2 Wong YY, McCaul KA, Yeap BB, Hankey GJ \& Flicker L. Low vitamin $\mathrm{D}$ status is an independent predictor of increased frailty and all-cause mortality in older men: the Health in Men Study. Journal of Clinical Endocrinology and Metabolism 201398 3821-3828. (https://doi. org/10.1210/jc.2013-1702)

3 Tague SE, Clarke GL, Winter MK, McCarson KE, Wright DE \& Smith PG. Vitamin D deficiency promotes skeletal muscle hypersensitivity and sensory hyperinnervation. Journal of Neuroscience 201131 13728-13738. (https://doi.org/10.1523/ JNEUROSCI.3637-11.2011)

4 Jesus CA, Feder D \& Peres MF. The role of vitamin D in pathophysiology and treatment of fibromyalgia. Current Pain and Headache Reports 201317 355. (https://doi.org/10.1007/s11916-0130355-6)

5 Mergenhagen K, Ott M, Heckman K, Rubin LM \& Kellick K. Low vitamin $\mathrm{D}$ as a risk factor for the development of myalgia in patients taking high-dose simvastatin: a retrospective review. Clinical Therapeutics 201436 770-777. (https://doi.org/10.1016/j. clinthera.2014.02.023)

6 Subash Shantha G, Ramos J, Thomas-Hemak L \& Pancholy SB. Association of vitamin D and incident statin induced myalgia - a retrospective cohort study. PLoS ONE 20149 e88877. (https://doi. org/10.1371/journal.pone.0088877)

7 Ceglia L, Niramitmahapanya S, da Silva Morais M, Rivas DA, Harris SS, Bischoff-Ferrari H, Fielding RA \& Dawson-Hughes B. A randomized study on the effect of vitamin $\mathrm{D}(3)$ supplementation on skeletal muscle morphology and vitamin D receptor concentration in older women. Journal of Clinical Endocrinology and Metabolism 2013 98 E1927-E1935. (https://doi.org/10.1210/jc.2013-2820)

8 McCarthy EK \& Kiely M. Vitamin D and muscle strength throughout the life course: a review of epidemiological and intervention studies. Journal of Human Nutrition and Dietetics 201528 636-645. (https:// doi.org/10.1111/jhn.12268)

9 Broe KE, Chen TC, Weinberg J, Bischoff-Ferrari HA, Holick MF $\&$ Kiel DP. A higher dose of vitamin D reduces the risk of falls in nursing home residents: a randomized, multiple-dose study. Journal of the American Geriatrics Society 200755 234-239. (https://doi. org/10.1111/j.1532-5415.2007.01048.x)

10 Lips P, Graafmans WC, Ooms ME, Bezemer PD \& Bouter LM. Vitamin $\mathrm{D}$ supplementation and fracture incidence in elderly persons. A randomized, placebo-controlled clinical trial. Annals of Internal Medicine 1996124 400-406. (https://doi.org/10.7326/0003-4819124-4-199602150-00003)

11 Annweiler C, Beauchet O, Berrut G, Fantino B, Bonnefoy M, Herrmann FR \& Schott AM. Is there an association between serum 
25-hydroxyvitamin D concentration and muscle strength among older women? Results from baseline assessment of the EPIDOS study. Journal of Nutrition, Health and Aging 200913 90-95. (https://doi. org/10.1007/s12603-009-0013-1)

12 Ovesjo ML, Skilving I, Bergman P, Rane A, Ekstrom L \& BjorkhemBergman L. Low vitamin D levels and genetic polymorphism in the vitamin D receptor are associated with increased risk of statininduced myopathy. Basic and Clinical Pharmacology and Toxicology 2016118 214-218. (https://doi.org/10.1111/bcpt.12482)

13 Ramagopalan SV, Heger A, Berlanga AJ, Maugeri NJ, Lincoln MR, Burrell A, Handunnetthi L, Handel AE, Disanto G, Orton SM, et al. A ChIP-seq defined genome-wide map of vitamin $\mathrm{D}$ receptor binding: associations with disease and evolution. Genome Research 201020 1352-1360. (https://doi.org/10.1101/gr.107920.110)

14 Dusso AS, Brown AJ \& Slatopolsky E. Vitamin D. American Journal of Physiology: Renal Physiology 2005289 F8-F28. (https://doi. org/10.1152/ajprenal.00336.2004)

15 Nagpal S, Na S \& Rathnachalam R. Noncalcemic actions of vitamin D receptor ligands. Endocrine Reviews 200526 662-687. (https://doi. org/10.1210/er.2004-0002)

16 Olsson K, Saini A, Stromberg A, Alam S, Lilja M, Rullman E \& Gustafsson T. Evidence for vitamin D receptor expression and direct effects of 1alpha,25(OH)2D3 in human skeletal muscle precursor cells. Endocrinology 2016157 98-111. (https://doi.org/10.1210/ en.2015-1685)

17 Camperi A, Pin F, Costamagna D, Penna F, Menduina ML, Aversa Z, Zimmers T, Verzaro R, Fittipaldi R, Caretti G, et al. Vitamin D and VDR in cancer cachexia and muscle regeneration. Oncotarget 20178 21778-21793. (https://doi.org/10.18632/oncotarget.15583)

18 Wynn RL. Vitamin D deficiency and nonspecific musculoskeletal pain. General Dentistry 201361 10-13.

19 Hewison M. Antibacterial effects of vitamin D. Nature Reviews: Endocrinology 20117 337-345. (https://doi.org/10.1038/ nrendo.2010.226)

20 Wrzosek M, Lukaszkiewicz J, Wrzosek M, Jakubczyk A, Matsumoto H, Piatkiewicz P, Radziwon-Zaleska M, Wojnar M \& Nowicka G. Vitamin $\mathrm{D}$ and the central nervous system. Pharmacological Reports $2013 \mathbf{6 5}$ 271-278. (https://doi.org/10.1016/S1734-1140(13)71003-X)

21 Boland R. Role of vitamin D in skeletal muscle function. Endocrine Reviews 19867 434-448. (https://doi.org/10.1210/edrv-7-4-434)

22 Endo I, Inoue D, Mitsui T, Umaki Y, Akaike M, Yoshizawa T, Kato S \& Matsumoto T. Deletion of vitamin D receptor gene in mice results in abnormal skeletal muscle development with deregulated expression of myoregulatory transcription factors. Endocrinology $2003 \mathbf{1 4 4}$ 5138-5144. (https://doi.org/10.1210/en.2003-0502)

23 Charge SB \& Rudnicki MA. Cellular and molecular regulation of muscle regeneration. Physiological Reviews 200484 209-238. (https:// doi.org/10.1152/physrev.00019.2003)

24 Walsh S, Ludlow AT, Metter EJ, Ferrucci L \& Roth SM. Replication study of the vitamin D receptor (VDR) genotype association with skeletal muscle traits and sarcopenia. Aging Clinical and Experimental Research 201628 435-442. (https://doi.org/10.1007/s40520-015$0447-8)$

25 Bergman P, Norlin AC, Hansen S, Rekha RS, Agerberth B, Bjorkhem-Bergman L, Ekstrom L, Lindh JD \& Andersson J. Vitamin D3 supplementation in patients with frequent respiratory tract infections: a randomised and double-blind intervention study. BMJ Open 20122 e001663. (https://doi.org/10.1136/ bmjopen-2012-001663)

26 Saini A, Rullman E, Lilja M, Mandic M, Melin M, Olsson K \& Gustafsson T. Asymmetric cellular responses in primary human myoblasts using sera of different origin and specification. PLOS ONE 201813 e0192384. (https://doi.org/10.1371/journal.pone.0192384)

27 Bergström J. Muscle electrolytes in man. Scandinavian Journal of Clinical and Laboratory Investigation 196214 1-110.
28 Park YG, Moon JH \& Kim J. A comparative study of magneticactivated cell sorting, cytotoxicity and preplating for the purification of human myoblasts. Yonsei Medical Journal 200647 179-183. (https://doi.org/10.3349/ymj.2006.47.2.179)

29 Carpenter AE, Jones TR, Lamprecht MR, Clarke C, Kang IH, Friman O, Guertin DA, Chang JH, Lindquist RA, Moffat J, et al. CellProfiler: image analysis software for identifying and quantifying cell phenotypes. Genome Biology 20067 R100. (https://doi. org/10.1186/gb-2006-7-10-r100)

30 Chen Y, Lun ATL \& Smyth GK. Differential expression analysis of complex RNA-seq experiments using edgeR. In Statistical Analysis of Next Generation Sequence Data. Ed S Nettleton. New York, NY, USA: Springer, 2014. (https://doi.org/10.1007/978-3-319-07212-8_3)

31 van der Maaten L. Accelerating t-SNE using tree-based algorithms. Journal of Machine Learning Research 201415 3221-3245. (available at: http://jmlr.org/papers/volume15/vandermaaten14a/ vandermaaten14a.pdf)

32 Yevshin I, Sharipov R, Valeev T, Kel A \& Kolpakov F. GTRD: a database of transcription factor binding sites identified by ChIP-seq experiments. Nucleic Acids Research 201745 D61-D67. (https://doi. org/10.1093/nar/gkw951)

33 Gopinath SD, Webb AE, Brunet A \& Rando TA. FOXO3 promotes quiescence in adult muscle stem cells during the process of selfrenewal. Stem Cell Reports 20142 414-426. (https://doi.org/10.1016/j. stemcr.2014.02.002)

34 Horsley V, Jansen KM, Mills ST \& Pavlath GK. IL-4 acts as a myoblast recruitment factor during mammalian muscle growth. Cell $2003 \mathbf{1 1 3}$ 483-494. (https://doi.org/10.1016/S0092-8674(03)00319-2)

35 Horsley V \& Pavlath GK. NFAT: ubiquitous regulator of cell differentiation and adaptation. Journal of Cell Biology 2002156 771-774. (https://doi.org/10.1083/jcb.200111073)

36 Liu N, Nelson BR, Bezprozvannaya S, Shelton JM, Richardson JA, Bassel-Duby R \& Olson EN. Requirement of MEF2A, C, and D for skeletal muscle regeneration. PNAS 2014111 4109-4114. (https:// doi.org/10.1073/pnas.1401732111)

37 Olsson K, Cheng AJ, Alam S, Al-Ameri M, Rullman E, Westerblad H, Lanner JT, Bruton JD \& Gustafsson T. Intracellular Ca(2+)-handling differs markedly between intact human muscle fibers and myotubes. Skeletal Muscle 20155 26. (https://doi.org/10.1186/s13395-015-0050-x)

38 Whitfield GK, Remus LS, Jurutka PW, Zitzer H, Oza AK, Dang HT, Haussler CA, Galligan MA, Thatcher ML, Encinas Dominguez C, et al. Functionally relevant polymorphisms in the human nuclear vitamin D receptor gene. Molecular and Cellular Endocrinology 2001177 145-159. (https://doi.org/10.1016/S0303-7207(01)00406-3)

39 McFarlane C, Hui GZ, Amanda WZ, Lau HY, Lokireddy S, Xiaojia G, Mouly V, Butler-Browne G, Gluckman PD, Sharma M, et al. Human myostatin negatively regulates human myoblast growth and differentiation. American Journal of Physiology: Cell Physiology 2011 301 C195-C203. (https://doi.org/10.1152/ajpcell.00012.2011)

40 Langley B, Thomas M, Bishop A, Sharma M, Gilmour S \& Kambadur R. Myostatin inhibits myoblast differentiation by downregulating MyoD expression. Journal of Biological Chemistry 2002277 49831-49840. (https://doi.org/10.1074/jbc.M204291200)

41 Thomas M, Langley B, Berry C, Sharma M, Kirk S, Bass J \& Kambadur R. Myostatin, a negative regulator of muscle growth, functions by inhibiting myoblast proliferation. Journal of Biological Chemistry 2000275 40235-40243. (https://doi.org/10.1074/jbc. M004356200)

42 Garcia LA, King KK, Ferrini MG, Norris KC \& Artaza JN. 1,25(OH)2 vitamin D3 stimulates myogenic differentiation by inhibiting cell proliferation and modulating the expression of promyogenic growth factors and myostatin in C2C12 skeletal muscle cells. Endocrinology 2011152 2976-2986. (https://doi.org/10.1210/en.2011-0159)

43 Girgis CM, Clifton-Bligh RJ, Mokbel N, Cheng K \& Gunton JE. Vitamin D signaling regulates proliferation, differentiation, and https://ec.bioscientifica.com https://doi.org/10.1530/EC-19-0194 (c) 2019 The authors Published by Bioscientifica Ltd

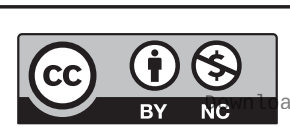

This work is licensed under a Creative Commons Attribution-NonCommercial 4.0 International License. ded from Bioscientifica.com at 04/26/2023 11:01:33AM 
myotube size in C2C12 skeletal muscle cells. Endocrinology 2014155 347-357. (https://doi.org/10.1210/en.2013-1205)

44 Braga M, Simmons Z, Norris KC, Ferrini MG \& Artaza JN. Vitamin $\mathrm{D}$ induces myogenic differentiation in skeletal muscle derived stem cells. Endocrine Connections 20176 139-150. (https://doi.org/10.1530/ EC-17-0008)

45 Tierney MT, Stec MJ, Rulands S, Simons BD \& Sacco A. Muscle stem cells exhibit distinct clonal dynamics in response to tissue repair and homeostatic aging. Cell Stem Cell 201822 119.e113-127.e113. (https://doi.org/10.1016/j.stem.2017.11.009)

46 Carlson ME, Conboy MJ, Hsu M, Barchas L, Jeong J, Agrawal A, Mikels AJ, Agrawal S, Schaffer DV \& Conboy IM. Relative roles of TGF-beta 1 and Wnt in the systemic regulation and aging of satellite cell responses. Aging Cell 20098 676-689. (https://doi.org/10.1111/ j.1474-9726.2009.00517.x)

47 Suetta C, Frandsen U, Mackey AL, Jensen L, Hvid LG, Bayer ML, Petersson SJ, Schroder HD, Andersen JL, Aagaard P, et al. Ageing is associated with diminished muscle re-growth and myogenic precursor cell expansion early after immobility-induced atrophy in human skeletal muscle. Journal of Physiology 2013591 3789-3804. (https://doi.org/10.1113/jphysiol.2013.257121)

48 Martineau AR, Timms PM, Bothamley GH, Hanifa Y, Islam K, Claxton AP, Packe GE, Moore-Gillon JC, Darmalingam M,

Davidson RN, et al. High-dose vitamin D(3) during intensive-phase antimicrobial treatment of pulmonary tuberculosis: a double-blind randomised controlled trial. Lancet 2011377 242-250. (https://doi. org/10.1016/S0140-6736(10)61889-2)

49 Selvaraj P, Chandra G, Jawahar MS, Rani MV, Rajeshwari DN \& Narayanan PR. Regulatory role of vitamin D receptor gene variants of Bsm I, Apa I, Taq I, and Fok I polymorphisms on macrophage phagocytosis and lymphoproliferative response to mycobacterium tuberculosis antigen in pulmonary tuberculosis. Journal of Clinical Immunology 200424 523-532. (https://doi.org/10.1023/ B:JOCI.0000040923.07879.31)

50 Okuno H, Kishimoto KN, Hatori M \& Itoi E. 1alpha,25Dihydroxyvitamin $\mathrm{D}(3)$ enhances fast-myosin heavy chain expression in differentiated C2C12 myoblasts. Cell Biology International 201236 441-447. (https://doi.org/10.1042/CBI20100782)

Received in final form 24 May 2019

Accepted 27 June 2019

Accepted Preprint published online 28 June 2019
This work is licensed under a Creative Commons Attribution-NonCommercial 4.0 International License. ded from Bioscientifica.com at 04/26/2023 11:01:33AM 